\title{
Neural Correlates of Forward and Inverse Models for Eye Movements: Evidence from Three-Dimensional Kinematics
}

\author{
Fatema F. Ghasia, Hui Meng, and Dora E. Angelaki \\ Department of Neurobiology, Washington University School of Medicine, St. Louis, Missouri 63110
}

Inverse and forward dynamic models have been conceptually important in computational motor control. In particular, inverse models are thought to convert desired action into appropriate motor commands. In parallel, forward models predict the consequences of the motor command on behavior by constructing an efference copy of the actual movement. Despite theoretical appeal, their neural representation has remained elusive. Here, we provide evidence supporting the notion that a group of premotor neurons called burst-tonic (BT) cells represent the output of the inverse model for eye movements. We show that BT neurons, like extraocular motoneurons but different from the evoked eye movement, do not carry signals appropriate for the half-angle rule of ocular kinematics during smoothpursuit eye movements from eccentric positions. Along with findings of identical response dynamics as motoneurons, these results strongly suggest that BT cells carry a replica of the motor command. In contrast, eye-head (EH) neurons, a premotor cell type that is the target of Purkinje cell inhibition from the cerebellar flocculus/ventral paraflocculus, exhibit properties that could be consistent with the half-angle rule. Therefore, EH cells may be functionally related to the output of a forward internal model thought to construct an efference copy of the actual eye movement.

Key words: torsion; Listing's law; half-angle rule; burst-tonic cells; internal model; smooth pursuit

\section{Introduction}

A contemporary concept in motor control is that desired action is transformed into an appropriate motor command through an "inverse model" (called inverse, because it represents the inverse transformation of that performed by the motor plant) (Fig. 1). In parallel, comparison between desired and actual movement uses a "forward model" that predicts the consequences of the motor command on behavior (Shadmehr and Mussa-Ivaldi, 1994; Wolpert and Kawato, 1998). Despite strong evidence from computational, behavioral, and imaging studies, whether and how these concepts are represented in neural responses has been sketchy (Imamizu et al., 2003; Zago et al., 2004; Pasalar et al., 2006).

Accordingly, desired eye velocity must be processed by an inverse model of the eye plant. Although motoneurons are restricted to innervating the extraocular muscles, a replica of such a signal must be fed into a forward model to predict the actually evoked eye movement (Fig. 1, feedback loop). Therefore, for the brain to have access to the motor command, the inverse model output must also be explicitly represented in dedicated neural populations other than motoneurons. Superb candidates for this role are burst-tonic (BT) neurons that are found throughout the brainstem, including the nucleus prepositus hypoglossi (McFar-

Received Feb. 4, 2008; revised March 12, 2008; accepted April 6, 2008.

This work was supported by National Institutes of Health Grant R01 EY15271. We thank Shoji Watanabe for his participation in neural recordings from the interstitial nucleus of Cajal, as well as Eliana Klier for comments on this manuscript.

Correspondence should be addressed to Dr. Dora Angelaki, Department of Anatomy and Neurobiology, Box 8108 Washington University School of Medicine, 660 South Euclid Avenue, St. Louis, M0 63110. E-mail: angelaki@pcg.wustl.edu.

DOI:10.1523/JNEUROSCI.0513-08.2008

Copyright $\odot 2008$ Society for Neuroscience $\quad$ 0270-6474/08/285082-06\$15.00/0 land and Fuchs, 1992), vestibular nuclei (Scudder and Fuchs, 1992), interstitial nucleus of Cajal (Fukushima, 1991), and paramedian tract (Buttner-Ennever and Horn, 1996; Nakamagoe et al., 2000). The notion that BT cells represent the output of the inverse model was first introduced by Belknap and McCrea (1988) and recently resurrected, because BT cell dynamics during pursuit and the vestibulo-ocular reflex (VOR) are identical to those of extraocular motoneurons (Green et al., 2007).

The present study was designed to further test this hypothesis. The focus here is on three-dimensional (3D) ocular kinematics. In particular, visually guided eye movements initiated from eccentric positions have rotation axes that do not remain confined to Listing's plane (zero torsion) but tilt torsionally in the same direction as gaze by approximately half as much (known as the half-angle rule) (Tweed and Vilis, 1990; Crawford and Vilis, 1991). This property appears to be generated by the mechanical characteristics of the eyeball, likely an eye position dependence of the extraocular muscle pulling directions brought about by orbital "pulleys" (Miller, 1989; Demer et al., 2000; Kono et al., 2002; Klier et al., 2006). Therefore, unlike the evoked eye movement, motoneurons do not encode the half-angle rule (Ghasia and Angelaki, 2005).

This difference between motor command and eye movement provides an ideal forum for testing whether specific cell types constitute neural substrates for the inverse or forward model. Specifically, if BT neurons represent the inverse model output and carry a replica of the motoneuron signal, they should exhibit no systematic eye position dependence consistent with the halfangle rule. In contrast, neurons representing the forward model (i.e., those that carry an efference copy of the actual eye move- 


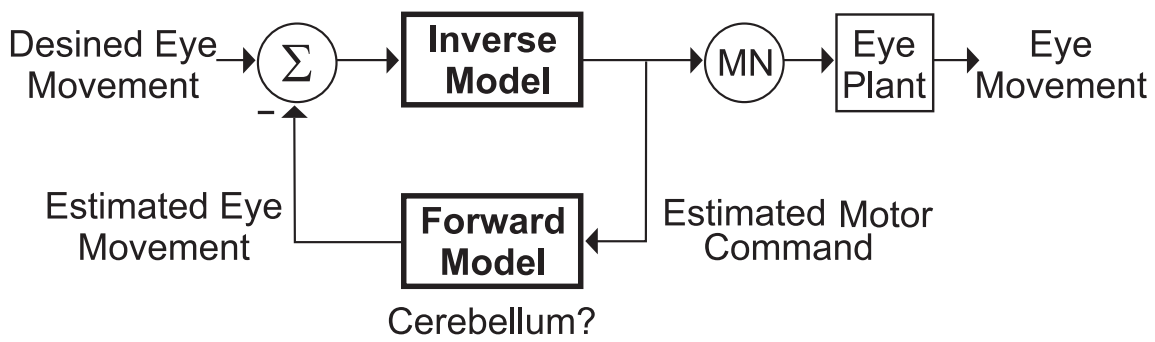

Figure 1. Schematic of inverse and forward models for eye movement generation. In addition to driving motoneurons (MN) and the eye plant, an efference copy of the motor command is also used by a forward model to compute an efference copy of the evoked eye movement, a signal needed to refine the motor command [adapted from the study by Green et al. (2007)].

ment) should exhibit a systematic eye position dependence that parallels that of the eye movement.

\section{Materials and Methods}

Data were collected from two Maccaca fascicularis and three Maccaca mulatta. 3D eye movements $(833.33 \mathrm{~Hz})$ were calibrated daily while fixating vertically and horizontally eccentric targets (Klier et al., 2005). Electrode penetrations were made into the nucleus prepositus hypoglossi, the medial and superior vestibular nuclei [same neurons as in the studies by Green et al. (2007), Meng et al. (2005), and Meng and Angelaki (2006)], and the interstitial nucleus of Cajal, as well as cells scattered between the abducens and oculomotor nuclei [locations identified in the studies by Ghasia and Angelaki (2005) and Klier et al. (2006)].

We tested premotor cells with the same experimental protocol that was used for extraocular motoneuron recordings (Ghasia and Angelaki, 2005). Each cell was first classified as BT, eye-head (EH), or positionvestibular-pause (PVP) based on its responses during horizontal/vertical fixations and pursuit, as well as sinusoidal horizontal (yaw) and vertical (pitch) rotations $\left(0.5 \mathrm{~Hz}, \pm 10^{\circ}\right)$ while fixating a central, head-fixed target (i.e., VOR cancellation). The main experimental protocol consisted of $0.6 \mathrm{~Hz}\left( \pm 5^{\circ} ; \sim 20^{\circ} / \mathrm{s}\right)$ horizontal pursuit with the eyes at $5-7$ vertical eccentricities and vertical pursuit at corresponding horizontal eccentricities $\left(0^{\circ}, \pm 10^{\circ}, \pm 15^{\circ}\right.$, and $\left.\pm 20^{\circ}\right)$. In a subpopulation of neurons, cell activity was also recorded during roll VOR $\left(2 \mathrm{~Hz} ; \pm 1.2^{\circ}\right)$ while the monkey maintained fixation of a central target.

$3 \mathrm{D}$ eye positions were expressed as rotation vectors using straight ahead as the reference position. Eye movements were expressed in a right hand coordinate system with positive directions being clockwise, downward, or leftward from the subject's viewpoint. Saccades and fast phases were identified and removed using a semiautomated algorithm based on higher derivatives of eye velocity, followed by manual verification. Neural data, expressed as instantaneous firing rate, were also "desaccaded" using a window that extended from $50 \mathrm{~ms}$ before to $200 \mathrm{~ms}$ after each saccade.

Portions of neural activity in which eye position was within $\pm 2^{\circ}$ of the target was folded into a single-cycle instantaneous frequency response and quantified (along with the horizontal, vertical, and torsional components of eye velocity) by fitting a sine function (Angelaki and Dickman, 2003; Ghasia and Angelaki, 2005). Response "gains" were expressed as spikes per second per degree per second of evoked eye velocity by dividing peak-to-trough firing rate with peak-to-trough horizontal, vertical, or torsional eye velocity.

To quantitatively compare with the findings in motoneurons, we followed an identical analysis to that performed by Ghasia and Angelaki (2005). Briefly, we plotted neural response gains as a function of target eccentricity and quantified these relationships using linear regression. This was first done separately for horizontal and vertical pursuit. In addition, we also simultaneously fitted the horizontal and vertical pursuit dependence of each cell on eye position, forcing them to have the same slope. We then compared these actual slopes with "predicted" slopes, computed from roll VOR responses (Ghasia and Angelaki, 2005). Expected slopes were computed as the product of two terms: the slope of the eye velocity dependence on target eccentricity (measured separately for each animal) and the sensitivity of the cell to torsional eye velocity (in units of spikes/second per degree/second), computed from the roll VOR. Notice that in this analysis, the sign of the predicted slopes (i.e., whether firing rates should increase or decrease as a function of eye position) was dependent on whether the neuron preferred clockwise or counter-clockwise torsion.

\section{Results}

We recorded responses to horizontal/vertical pursuit in 125 eye movement-sensitive premotor neurons in the prepositus/vestibular nuclei (including superior vestibular nuclei and y-group; $n=73$ ), the interstitial nucleus of Cajal $(n=16)$, and the perioculomotor region, including the area between the oculomotor and abducens nuclei known as the paramedian tract $(n=36)$. On the basis of their discharge properties during smooth pursuit and the VOR while fixating a head-fixed target (VOR cancellation), neurons were classified into three groups: $\mathrm{EH}(n=46)$, PVP $(n=$ $11)$, and BT $(n=80)$ cells (Scudder and Fuchs, 1992). PVP and $\mathrm{EH}$ cells were restricted to the vestibular nuclei, whereas BT neurons were encountered in all areas. Because there was no difference in BT cell responses in the prepositus/vestibular nuclei, nucleus of Cajal, and perioculomotor regions, data have been grouped together.

The main protocol consisted of horizontal pursuit at different vertical eccentricities and vertical pursuit at different horizontal eccentricities, as illustrated for an EH cell in Figure 2, $A$ and $B$. During horizontal pursuit at zero vertical eccentricity (central target), the animal pursued the target with an almost purely horizontal eye velocity (Fig. $2 \mathrm{~A}$, middle). In contrast, during horizontal pursuit in vertically eccentric positions, eye velocity had both horizontal and torsional components, the latter being opposite in direction for up versus down targets (Fig. $2 \mathrm{~A}$, left and right panels; for easier visualization, vertical dotted lines mark the relative troughs and peaks of horizontal and torsional eye velocity). Therefore, when eye velocity was plotted in head coordinates (Fig. 2A, left inset with monkey drawing), eye velocity tilted in the same direction as gaze by approximately half as much (accounting for the half-angle rule). This torsional component of eye velocity is necessary to keep eye position in Listing's plane (Tweed and Vilis, 1990). Similar observations were made for vertical pursuit (Fig. $2 B$ ). The eye velocity tilted away from a purely horizontal or purely vertical direction in proportion to orthogonal eye position and was quantified in each animal using linear regression (Fig. 2, left inset, black solid lines fitted to eye position data) (Ghasia and Angelaki, 2005).

The goal of this study was to examine whether premotor neurons change their activity to reflect the added torsional velocity (and thus the tilt of eye velocity) during eccentric pursuit. If they are motoneuron-like, they should not (Ghasia and Angelaki, 2005). Alternatively, premotor neurons might change their firing rates with pursuit eccentricity in proportion to their sensitivity to torsional eye movements (which can be directly measured during the roll VOR). To quantitatively characterize the eye position dependence of pursuit modulation of premotor neurons, like the EH cell in Figure $2(A$ and $B$ ), we computed response gains, which were then plotted as a function of target eccentricity (Fig. 2C,D). The resulting slope describing how much peak firing rate changes as a function of eye position was quantified using linear regression. We first did this analysis by fitting the data separately for horizontal and vertical pursuit responses (Fig. 2C,D, slopes of 
0.022 and 0.0056 , respectively). Figure 3 compares scatter plots of the horizontal versus vertical pursuit slope magnitude for $\mathrm{BT}, \mathrm{EH}$, and PVP neurons, respectively, with the corresponding data from extraocular motoneurons (Ghasia and Angelaki, 2005). There was no difference in either horizontal or vertical pursuit slopes between BT neurons and cyclovertical motoneurons (Kruskal-Wallis test; $p \gg$ 0.05), and neither was there any significant difference between PVP and motoneuron slopes (Kruskal-Wallis test; $p \gg 0.05$ ). However, EH cells tended to have higher slopes than extraocular motoneurons during horizontal pursuit (Kruskal-Wallis test; $p=0.03$ ) and vertical pursuit (Kruskal-Wallis test; $p=0.06$ ).

For a subpopulation of cells, responses were also obtained during roll rotation. For these neurons, it was possible to compute a "predicted" eye position dependence that was proportional to the respective sensitivity of the cell to torsional eye movements (see Materials and Methods) (Ghasia and Angelaki, 2005). Figure 4 compares the actual with the predicted slopes for BT and EH cells, with sensitivities to torsional eye velocity that were directly estimated during $2 \mathrm{~Hz}$ roll head movements (PVP neurons were not tested during roll VOR). Although there was no significant difference between predicted and actual values for BT cells (Wilcoxon matched pairs test; $p=$ $0.65)$, the two values were not correlated (Spearman rank correlation; $r=-0.08$; $p=0.73$ ) (Fig. 4, open triangles). Notably, changes in BT firing rates were often in the incorrect direction from that predicted based on the torsional on-direction of the cell). Therefore, like motoneurons (Ghasia and Angelaki, 2005), the eye position dependence of BT neuron firing rates was small and often in the wrong direction from that predicted if these cells encoded the half-angle rule (Fig. 4 , gray areas). Notably, BT and EH cells appeared to behave differently (Fig. 4, compare open triangles and filled squares). EH cells showed a clear correlation between predicted and actual eye position dependencies (Spearman rank correlation; $r=0.76 ; p=$ 0.01 ), with a slope that was not different from unity (slope, [ $95 \%$ $\mathrm{CI}]=1.43[0.57,2.31])$. The two values were also not statistically different from each other (Wilcoxon matched pairs test; $p=$ 0.11). Therefore, unlike BT cells, EH neuron firing rates might be consistent with coding of the half-angle rule. Similar conclusions were also reached from a larger sample of EH cells (Angelaki and Dickman, 2003).

\section{Discussion}

\section{Inverse model and BT neurons}

To generate a movement (e.g., of the eye), the brain generates appropriate motor commands through an inverse model (Fig. 1) (called inverse, because it represents the inverse transformation of that performed by the motor plant). The inverse model con-
Horizontal pursuit

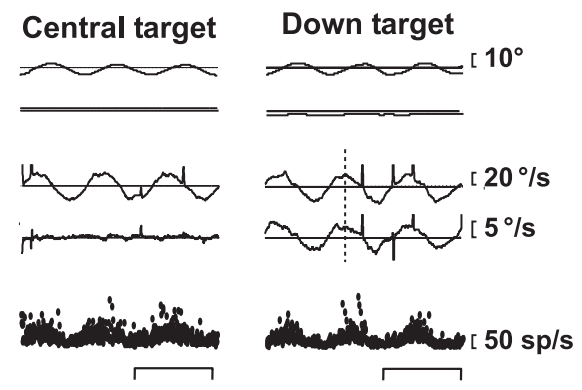

Vertical pursuit
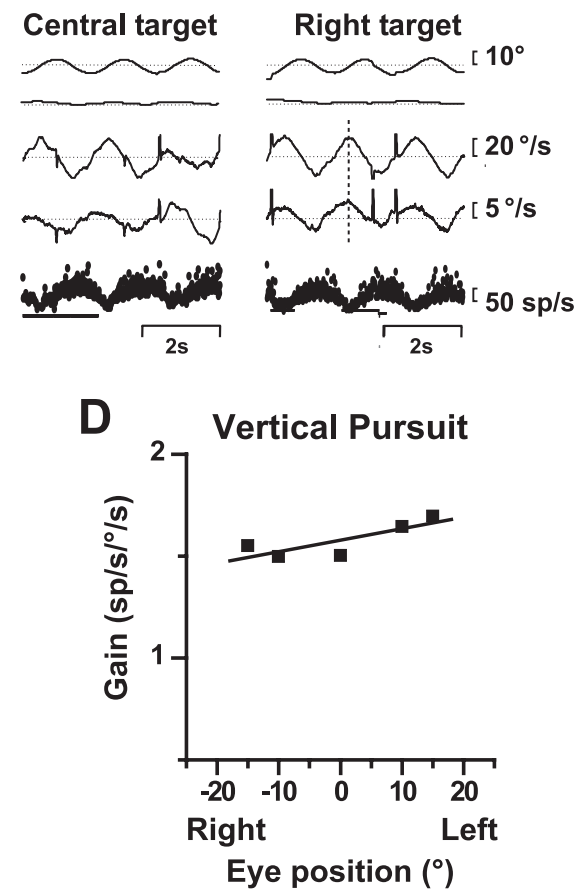

Figure 2. $\quad \boldsymbol{A}, \boldsymbol{B}$, Eye position (Pos), eye velocity (Vel), and neural responses from an EH neuron during horizontal (Hor) pursuit $(\boldsymbol{A})\left[15^{\circ}\right.$ up, center, and $20^{\circ}$ down $\left.(\mathrm{dn})\right]$ and vertical (Ver) pursuit $(\boldsymbol{B})\left[15^{\circ}\right.$ left $(\mathrm{L})$ center, and $15^{\circ}$ right $\left.(\mathrm{R})\right]$. IFR, Instantaneous fote; Tor, torsion. Insets, Eye velocity in head coordinates (monkey drawing). Data are shown in gray, with superimposed black solid lines illustrating linear regression (shown for eccentric targets). C, D, Response gain versus eye position for horizontal and vertical pursuit. Solid lines, Linear regression.

cept in the oculomotor system was pioneered by Skavenski and Robinson (1973). How this inverse model is implemented by the brain is, however, less straight forward. Obviously, the original description in terms of two parallel but independent pathways, one coding eye velocity and the other its integral (eye position), is clearly an oversimplification. On one hand, premotor neurons are heavily interconnected with each other. In addition, all neuron types carry signals related to both eye velocity and eye position (McFarland and Fuchs, 1992; Scudder and Fuchs, 1992). Therefore, exactly how the inverse model for eye movements is generated continues to be a mystery (Green et al., 2007).

Because of delays in sensory/proprioceptive feedback, computational models also require that an efference copy of the motor command is available to the brain, such that it can be used by forward models to predict the consequences of the motor command on behavior (i.e., to compute an efference copy of the movement itself) (Wolpert and Kawato, 1998). Because motoneurons are restricted to innervating the extraocular muscles, the output of the inverse model is also represented in BT cells, 

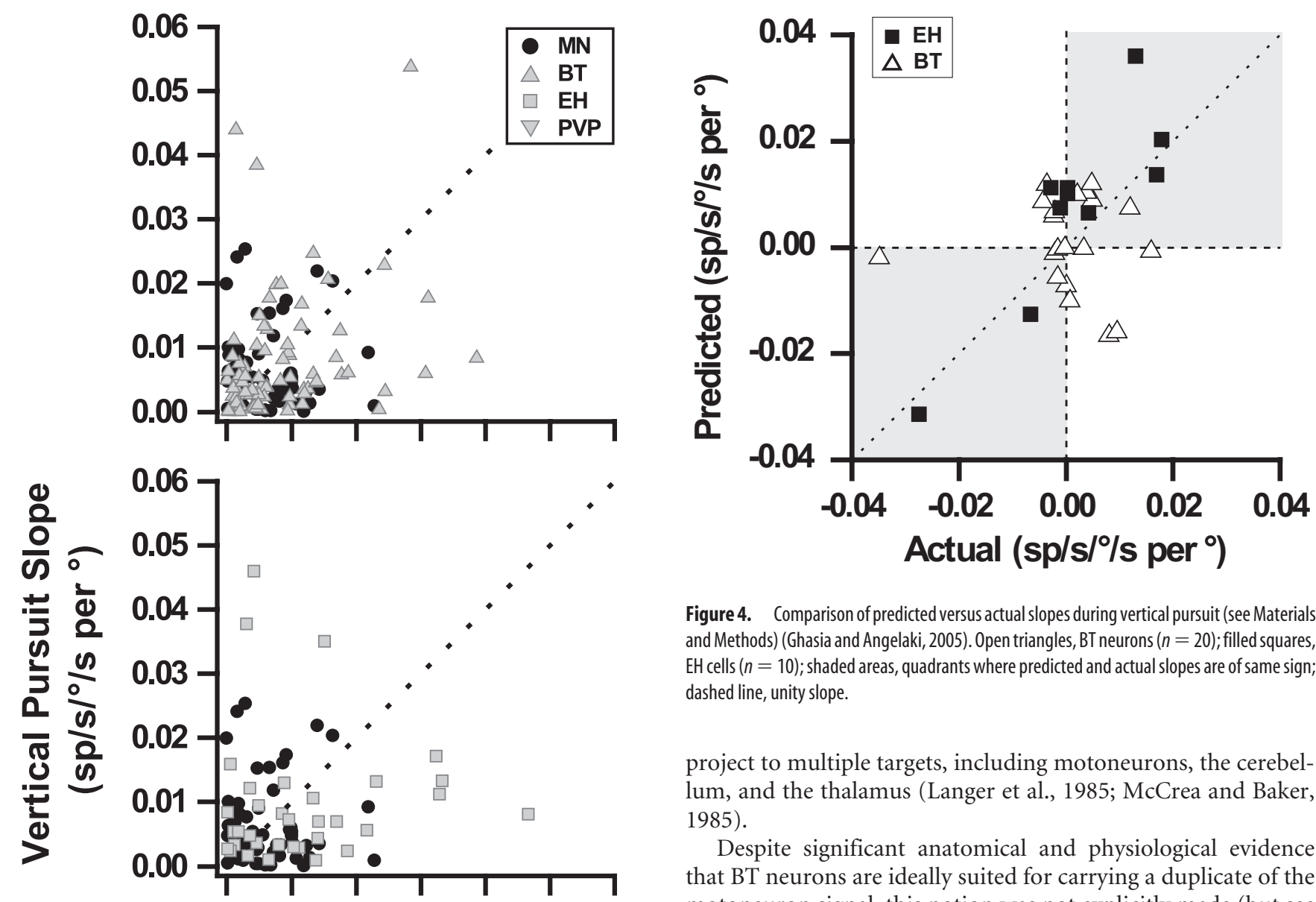

Figure 4. Comparison of predicted versus actual slopes during vertical pursuit (see Materials and Methods) (Ghasia and Angelaki, 2005). Open triangles, BT neurons ( $n=20)$; filled squares, EH cells $(n=10)$; shaded areas, quadrants where predicted and actual slopes are of same sign; dashed line, unity slope.

project to multiple targets, including motoneurons, the cerebellum, and the thalamus (Langer et al., 1985; McCrea and Baker, 1985).

Despite significant anatomical and physiological evidence that BT neurons are ideally suited for carrying a duplicate of the motoneuron signal, this notion was not explicitly made (but see Belknap and McCrea, 1988) until very recently when BT neurons during pursuit and the VOR were shown to have identical dynamics to those of extraocular motoneurons (Green et al., 2007). In particular, by comparing the high-frequency responses of horizontal BT neurons and motoneurons, Green et al. (2007) proposed that, like motoneurons, BT cells represent the output of the inverse model. Here, we provide strong support for this hypothesis by also demonstrating that, like motoneurons, BT neurons do not appear to encode the half-angle rule of 3D ocular kinematics during pursuit eye movements (see below). Therefore, unlike motoneurons with a job that is to produce the movement, the primary function of BT cells is to distribute an efference copy of the motor command elsewhere in the brain (McCrea and Baker, 1985; Belknap and McCrea, 1988).

The half-angle rule and 3D ocular kinematics

The present experiments were motivated by recent findings that motoneuron discharge (reflecting the motor command) and the eye movement being generated differ in their 3D ocular kinematics. In particular, for eye positions to remain in Listing's plane, the axis of eye rotation during horizontal and vertical pursuit eye movements initiated from eccentric positions must tilt out of Listing's plane, in the same direction as gaze, by approximately half as much (half-angle rule) (Tweed and Vilis, 1990). In contrast, motoneurons show no evidence for coding this eye position dependence of eye velocity (Ghasia and Angelaki, 2005). Instead, the half-angle rule appears to be implemented by the mechanical properties of the eyeball: likely, orbital pulleys make the pulling directions of extraocular muscles eye position dependent (Miller, 1989; Demer et al., 2000; Kono et al., 2002; Klier et al., 2006).

Notably, the brain must have access to these 3D eye movement 
kinematics, even if motoneurons do not (for review, see Crawford et al., 2003; Angelaki and Hess, 2004). At least three experimental findings argue for this. First, different eye movements exhibit different 3D kinematics. Saccades, pursuit, and the translational VOR follow Listing's law and the half-angle rule (Tweed and Vilis, 1990; Angelaki et al., 2003). However, Listing's law is relaxed or abandoned during the rotational VOR (Crawford and Vilis, 1991; Misslisch et al., 1994; Angelaki, 2003). Second, during head-free gaze shifts, where slower head movements are routinely preceded by an eye saccade, the saccade normally drives the eyes out of Listing's plane in an anticipatory manner, such that eye position ends up in Listing's plane after the whole movement is complete (Crawford et al., 2003). Third, the visuomotor transformations from retinal information into kinematically correct eye movements require accurate neural representation of $3 \mathrm{D}$ ocular kinematics (for review, see Crawford et al., 2003).

\section{Forward model and EH cells}

Therefore, despite an ocular plant that implements 3D ocular kinematics, there is clearly a need for the brain to have access to the half-angle rule. That is, to generate appropriate motor commands, particularly during more complicated eye-head coordination and visuomotor transformation tasks, a forward model must take into account the efference copy of the motor command (carried by BT neurons) to compute an efference copy of the evoked eye movement (Fig. 1). Therefore, unlike the inverse model, neurons implementing the forward model would be expected to code for the half-angle rule (because they should encode a neural estimate of the evoked eye movement). Such a signal can then be used to compute an error between predicted and desired action to help refine the motor command. This concept, which is well documented by contemporary theories of limb motor control, has been less influential in the oculomotor system (but see Glasauer, 2003; Green et al., 2007).

The cerebellum has long been implicated in eye movement adaptation and internal models (Shidara et al., 1993; Wolpert et al., 1995; Glasauer, 2003). EH neurons are the recipients of direct inhibition from Purkinje cells in the cerebellar flocculus and ventral paraflocculus and are shown to participate in motor learning (Lisberger et al., 1994; Zhang et al., 1995). Therefore, the present findings and those of Angelaki and Dickman (2003), showing that $\mathrm{EH}$ responses might be consistent with coding the half-angle rule during pursuit, provide strong support that the cerebellum might be constructing a forward model for slow eye movements. Such a forward model is important not only for refinement of the motor command and motor adaptation but also for appropriate $3 \mathrm{D}$ ocular kinematics. Indeed, the cerebellum is thought to provide the neural guidance over Listing's law and the half-angle rule (Helmchen et al., 1997; Straumann et al., 2000; Walker et al., 2007).

In summary, the present results relate to contemporary issues for eye movement generation that bring together the concept of internal models and recent controversies in neural versus mechanical control of $3 \mathrm{D}$ eye kinematics. Previous attempts searched for such neural signals consistent with 3D ocular kinematics in the premotor pathway for generating eye movements (van Opstal et al., 1991, 1996; Hepp et al., 1993; Scherberger et al., 2001). However, here, we show that the search should not be aimed at neural pathways that implement the inverse model (i.e., those directly involved in the generation of the movement), but rather at neural pathways that implement the forward model. Future studies should address further the role of the cerebellum and cerebellar-target neurons in the brainstem in relationship to these hypotheses.

\section{References}

Angelaki DE (2003) Three-dimensional ocular kinematics during eccentric rotations: evidence for functional rather than mechanical constraints. J Neurophysiol 89:2685-2696.

Angelaki DE, Dickman JD (2003) Premotor neurons encode torsional eye velocity during smooth-pursuit eye movements. J Neurosci 23:2971-2979.

Angelaki DE, Hess BJ (2004) Control of eye orientation: where does the brain's role end and the muscle's begin? Eur J Neurosci 19:1-10.

Angelaki DE, Zhou HH, Wei M (2003) Foveal versus full-field visual stabilization strategies for translational and rotational head movements. J Neurosci 23:1104-1108.

Belknap DB, McCrea RA (1988) Anatomical connections of the prepositus and abducens nuclei in the squirrel monkey. J Comp Neurol 268:13-28.

Buttner-Ennever JA, Horn AK (1996) Pathways from cell groups of the paramedian tracts to the floccular region. Ann NY Acad Sci 781:532-540.

Crawford JD, Vilis T (1991) Axes of eye rotation and Listing's law during rotations of the head. J Neurophysiol 65:407-423.

Crawford JD, Martinez-Trujillo JC, Klier EM (2003) Neural control of three-dimensional eye and head movements. Curr Opin Neurobiol 13:655-662.

Demer JL, Oh SY, Poukens V (2000) Evidence for active control of rectus extraocular muscle pulleys. Invest Ophthalmol Vis Sci 41:1280-1290.

Fukushima K (1991) The interstitial nucleus of Cajal in the midbrain reticular formation and vertical eye movement. Neurosci Res 10:159-187.

Ghasia FF, Angelaki DE (2005) Do motoneurons encode the noncommutativity of ocular rotations? Neuron 47:281-293.

Glasauer S (2003) Cerebellar contribution to saccades and gaze holding: a modeling approach. Ann NY Acad Sci 1004:206-219.

Green AM, Meng H, Angelaki DE (2007) A reevaluation of the inverse dynamic model for eye movements. J Neurosci 27:1346-1355.

Helmchen C, Glasauer S, Buttner U (1997) Pathological torsional eye deviation during voluntary saccades: a violation of Listing's law. J Neurol Neurosurg Psychiatry 62:253-260.

Hepp K, van Opstal AJ, Straumann D, Hess BJ, Henn V (1993) Monkey superior colliculus represents rapid eye movements in a two-dimensional motor map. J Neurophysiol 69:965-979.

Imamizu H, Kuroda T, Miyauchi S, Yoshioka T, Kawato M (2003) Modular organization of internal models of tools in the human cerebellum. Proc Natl Acad Sci USA 100:5461-5466.

Klier EM, Angelaki DE, Hess BJ (2005) Roles of gravitational cues and efference copy signals in the rotational updating of memory saccades. J Neurophysiol 94:468-478.

Klier EM, Meng H, Angelaki DE (2006) Three-dimensional kinematics at the level of the oculomotor plant. J Neurosci 26:2732-2737.

Kono R, Clark RA, Demer JL (2002) Active pulleys: magnetic resonance imaging of rectus muscle paths in tertiary gazes. Invest Ophthalmol Vis Sci 43:2179-2188.

Langer T, Fuchs AF, Scudder CA, Chubb MC (1985) Afferents to the flocculus of the cerebellum in the rhesus macaque as revealed by retrograde transport of horseradish peroxidase. J Comp Neurol 235:1-25.

Lisberger SG, Pavelko TA, Bronte-Stewart HM, Stone LS (1994) Responses during eye movements of brain stem neurons that receive monosynaptic inhibition from the flocculus and ventral paraflocculus in monkeys. J Neurophysiol 72:909-927.

McCrea RA, Baker R (1985) Anatomical connections of the nucleus prepositus of the cat. J Comp Neurol 237:377-407.

McFarland JL, Fuchs AF (1992) Discharge patterns in nucleus prepositus hypoglossi and adjacent medial vestibular nucleus during horizontal eye movement in behaving macaques. J Neurophysiol 68:319-332.

Meng H, Angelaki DE (2006) Neural correlates of the dependence of compensatory eye movements during translation on target distance and eccentricity. J Neurophysiol 95:2530-2540.

Meng H, Green AM, Dickman JD, Angelaki DE (2005) Pursuit-vestibular interactions in brain stem neurons during rotation and translation. J Neurophysiol 93:3418-3433.

Miller JM (1989) Functional anatomy of normal human rectus muscles. Vision Res 29:223-240.

Misslisch H, Tweed D, Fetter M, Sievering D, Koenig E (1994) Rotational 
kinematics of the human vestibuloocular reflex. III. Listing's law. J Neurophysiol 72:2490-2502.

Nakamagoe K, Iwamoto Y, Yoshida K (2000) Evidence for brainstem structures participating in oculomotor integration. Science 288:857-859.

Pasalar S, Roitman AV, Durfee WK, Ebner TJ (2006) Force field effects on cerebellar Purkinje cell discharge with implications for internal models. Nat Neurosci 9:1404-1411.

Scherberger H, Cabungcal JH, Hepp K, Suzuki Y, Straumann D, Henn V (2001) Ocular counterroll modulates the preferred direction of saccaderelated pontine burst neurons in the monkey. J Neurophysiol 86:935-949.

Scudder CA, Fuchs AF (1992) Physiological and behavioral identification of vestibular nucleus neurons mediating the horizontal vestibuloocular reflex in trained rhesus monkeys. J Neurophysiol 68:244-264.

Shadmehr R, Mussa-Ivaldi FA (1994) Adaptive representation of dynamics during learning of a motor task. J Neurosci 14:3208-3224.

Shidara M, Kawano K, Gomi H, Kawato M (1993) Inverse-dynamics model eye movement control by Purkinje cells in the cerebellum. Nature 365:50-52.

Skavenski AA, Robinson DA (1973) Role of abducens neurons in vestibuloocular reflex. J Neurophysiol 36:724-738.

Straumann D, Zee DS, Solomon D (2000) Three-dimensional kinematics of ocular drift in humans with cerebellar atrophy. J Neurophysiol 83:1125-1140.
Tweed D, Vilis T (1990) Geometric relations of eye position and velocity vectors during saccades. Vision Res 30:111-127.

van Opstal AJ, Hepp K, Hess BJ, Straumann D, Henn V (1991) Two- rather than three-dimensional representation of saccades in monkey superior colliculus. Science 252:1313-1315.

van Opstal J, Hepp K, Suzuki Y, Henn V (1996) Role of monkey nucleus reticularis tegmenti pontis in the stabilization of Listing's plane. J Neurosci 16:7284-7296.

Walker MF, Tian J, Zee DS (2007) Kinematics of the rotational vestibuloocular reflex: role of the cerebellum. J Neurophysiol 98:295-302.

Wolpert DM, Kawato M (1998) Multiple paired forward and inverse models for motor control. Neural Netw 11:1317-1329.

Wolpert DM, Ghahramani Z, Jordan MI (1995) An internal model for sensorimotor integration. Science 269:1880-1882.

Zago M, Bosco G, Maffei V, Iosa M, Ivanenko YP, Lacquaniti F (2004) Internal models of target motion: expected dynamics overrides measured kinematics in timing manual interceptions. J Neurophysiol 91:1620-1634.

Zhang Y, Partsalis AM, Highstein SM (1995) Properties of superior vestibular nucleus flocculus target neurons in the squirrel monkey. II. Signal components revealed by reversible flocculus inactivation. J Neurophysiol 73:2279-2292. 